\section{BRAZIULIAN JOURNAL \\ OF MEDICAL AND BIOLOGICAL RLSF.ARCH}

www.bjournal.com.br
ISSN 0100-879X

Volume 42 (11) 993-1118 November 2009

BIOMEDICAL SCIENCES

AND

CLINICAL INVESTIGATION

Braz J Med Biol Res, November 2009, Volume 42(11)1076-1079

Texture analysis of computed tomography images of acute ischemic stroke patients

M.S. Oliveira, P.T. Fernandes, W.M. Avelar, S.L.M. Santos, G. Castellano and L.M. Li

The Brazilian Journal of Medical and Biological Research is partially financed by
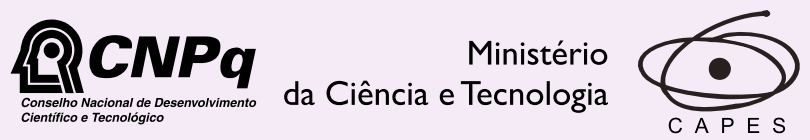

Ministério da Educação

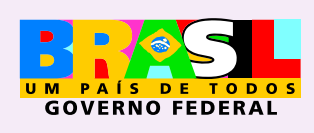

DFAPESP

Institutional Sponsors 


\title{
Texture analysis of computed tomography images of acute ischemic stroke patients
}

\author{
M.S. Oliveira ${ }^{1,3}$, P.T. Fernandes ${ }^{2,3}$, W.M. Avelar2,3, \\ S.L.M. Santos 2,3 , G. Castellano ${ }^{1,3}$ and L.M. Li ${ }^{2,3}$ \\ ${ }^{1}$ Grupo de Neurofísica, Departamento de Raios Cósmicos e Cronologia, Instituto de Física Gleb Wataghin, \\ ${ }^{2}$ Laboratório de Neuroimagem, Departamento de Neurologia, Faculdade de Ciências Médicas, \\ Universidade Estadual de Campinas, Campinas, SP, Brasil \\ 3Programa CInAPCe (Cooperação Interinstitucional de Apoio à Pesquisas sobre o Cérebro)
}

\begin{abstract}
Computed tomography (CT) images are routinely used to assess ischemic brain stroke in the acute phase. They can provide important clues about whether to treat the patient by thrombolysis with tissue plasminogen activator. However, in the acute phase, the lesions may be difficult to detect in the images using standard visual analysis. The objective of the present study was to determine if texture analysis techniques applied to CT images of stroke patients could differentiate between normal tissue and affected areas that usually go unperceived under visual analysis. We performed a pilot study in which texture analysis, based on the gray level co-occurrence matrix, was applied to the CT brain images of 5 patients and of 5 control subjects and the results were compared by discriminant analysis. Thirteen regions of interest, regarding areas that may be potentially affected by ischemic stroke, were selected for calculation of texture parameters. All regions of interest for all subjects were classified as lesional or non-lesional tissue by an expert neuroradiologist. Visual assessment of the discriminant analysis graphs showed differences in the values of texture parameters between patients and controls, and also between texture parameters for lesional and non-lesional tissue of the patients. This suggests that texture analysis can indeed be a useful tool to help neurologists in the early assessment of ischemic stroke and quantification of the extent of the affected areas.
\end{abstract}

Key words: Texture analysis; Computed tomography; Thrombolysis; Discriminant analysis

\section{Introduction}

Early assessment of brain stroke symptoms is fundamental to decide the prescription of thrombolysis treatment with tissue plasminogen activator (tPA), since several studies have demonstrated that this treatment may be effective only if applied within a window of 0 to $3 \mathrm{~h}$ after the onset of the stroke (1). Although studies have reported a 6 to $10 \%$ death rate for patients treated with tPA during hospitalization (2), the application of this treatment to acute ischemic stroke patients has a good prognosis for independent living after the therapy. Unfortunately, the recognition of stroke-affected areas in X-ray computed tomography (CT) images during the early hours of symptom onset can be difficult and highly dependent on the ability of the examiner $(3,4)$.

CT images are digital gray level images, i.e., they are formed by a rectangular array of pixels (picture elements), which can assume numerical values between 0 and $2^{\mathrm{N}-1}$ corresponding to gray levels, or different luminous intensi- ties. $\mathrm{N}$ is an integer, usually 12 , given that the $\mathrm{X}$-ray attenuation values for the CT voxels are normally expressed in Hounsfield units, which vary from -1000 to approximately 3000 . The human eye can discriminate between about 64 $(N=6)$ gray levels at a time (5), so that visual analysis can miss important information in a CT image. In fact, the human eye can distinguish many more than 64 gray levels due to the size adjustment of the pupil, but at one time, i.e., for a given pupil size, it can distinguish about 64 levels (5).

Our objective in this study was to perform a computational analysis, known as texture analysis, of the CT images of stroke patients in order to determine if this analysis could detect differences between lesioned and non-lesioned tissue that otherwise would go undetected.

Texture analysis is an important branch of digital image processing. Although a clear definition of texture does not exist, it can be understood to be a group of image properties

Correspondence: M.S. Oliveira, Grupo de Neurofísica, Departamento de Raios Cósmicos e Cronologia, Instituto de Física Gleb Wataghin, UNICAMP, Caixa Postal 6165, 13083-970 Campinas, SP, Brasil. Fax: +55-19-3521-5512. E-mail: marciaso@ifi.unicamp.br

M.S. Oliveira is supported by CNPq (\#141568/2005-4).

Received February 16, 2009. Accepted August 19, 2009. Available online October 13, 2009. 
that relate to our intuitive notions of coarseness, rugosity, smoothness, etc. (6). Many approaches have been developed to extract texture information from digital images and these may be grouped into four categories (7): 1) transform, 2) model-based, 3) structural, and 4) statistical techniques. Transform approaches comprise methods based on the Fourier or on the Wavelet transforms; they attempt to describe the image regions through their frequency content (Fourier) or their frequency and scale content (wavelets). In model-based methods, fractal models are used to describe texture. Structural techniques (6) use sets of primitive shapes to describe an object and include image texture assessment through mathematical morphology (7) operators. Last, statistical approaches (6) use the pixels gray level distribution to extract texture information from the image. This type of approach has been widely used to analyze medical images (8), and is quite suitable for this purpose since it does not rely on matching primitive forms, as do structural approaches. The statistical approach we adopted to extract texture parameters from CT images is based on the gray level co-occurrence (GLC) matrix.

Texture analysis based on statistical methods can be done using first- or second-order statistics. First-order statistical parameters measure the likelihood of a certain gray level being in a randomly chosen image location and depend only on the values of individual pixels. Secondorder statistics studies properties of gray level pairs. The method most frequently used to extract second-order statistical parameters from images is to compute the GLC matrix. The GLC matrix is an $N$ square matrix, where $N$ is the number of gray levels in the image. Each element $(i, j)$ of this matrix reports how many times gray level $i$ cooccurs with gray level $j$, given the distance (usually $d=1$, $2,3,4$, or 5 pixels) and the direction $\left(\theta=0^{\circ}, 45^{\circ}, 90^{\circ}\right.$, or $135^{\circ}$ ) between them.

After computing the GLC matrix the image can be analyzed according to Haralick's texture descriptors $(6,9)$. Haralick defined 14 descriptors, from which we selected 4 to perform our analysis:

Contrast: $C=\sum_{n=1}^{N} n^{2}\left[\sum_{i=1}^{N} \sum_{j=1}^{N} p(i, j)\right]_{|i-j|=n}$,

Angular second moment: $A S M=\sum_{i=1}^{N} \sum_{j=1}^{N}[p(i, j)]^{2}$,

Sum of squares: $S S=\sum_{i=1}^{N} \sum_{j=1}^{N}(i-\mu)^{2} p(i, j)$,

where $\mu=\sum_{i=1}^{N} \sum_{j=1}^{N} p(i, j)$, and
Sum variance: $S V=\sum_{i=2}^{2 N}\left(i-\sum_{k=2}^{2 N} k p_{x+y}(k)\right)^{2} p_{x+y}(i)$,

where $p_{x+y}(k)=\left[\sum_{i=1}^{N} \sum_{j=1}^{N} p(i, j)\right]_{i+j=k}$ and $k=2, \ldots, 2 N$.

In all equations, $p(i, j)$ is the $(i, j)$-th entry of the normalized GLC matrix, i.e., $p(i, j)=P(i, j) / \sum_{i j} P(i, j)$, where $P(i, j)$ is the $(i, j)$-th entry of the computed GLC matrix. $N$ is the total number of gray levels in the image.

Contrast refers to the relative difference between gray level values of different objects in the image. The angular second moment (ASM) gives information about the uniformity of the image. The sum of squares (SS) is similar to the variance, which indicates how much a measure differs from the mean. The sum variance (SV) does not have an intuitive definition and indicates how much the distribution of the sum probability $p_{x+y}(k)$ differs from its mean.

These descriptors were selected after computation of all the possible descriptors followed by inspection of their values and the 4 selected parameters were the ones that provided the highest differentiation amongst the different tissue types (normal and lesional).

\section{Material and Methods}

All CT images used were acquired with a General Electric CT scanner (USA; pixel size $0.5 \times 0.5 \mathrm{~mm}^{2}$ and 4096 gray levels). Subjects were 5 female patients with a clinical and image-confirmed diagnosis of ischemic brain stroke (4 with hemiparesis, 1 with mixed aphasia; mean age $53.00 \pm 8.06$ years) and 5 female controls ( 3 with migraine, 1 with transitory consciousness loss, 1 with headache; mean age $53.25 \pm$ 7.41 years). All images were analyzed by a radiologist and a neurologist who defined which regions of interest (ROIs) were normal and which were lesioned in the patients. The study was approved by the Ethics Committee of the University of Campinas, UNICAMP, Brazil, and all subjects gave written informed consent to participate.

For calculation of the texture parameters, $13 \mathrm{ROIs}$ were extracted from 2 slices from each subject and are illustrated in Figure 1. Ten of these ROls were selected according to the Alberta Stroke Program Early CT Score (ASPECTS) (10). ASPECTS was developed to assess the extent of vasculature involvement of the middle cerebral artery (MCA) in the process of ischemia. Basically, this method consists of assigning a point to each of 10 ROls affected by stroke, visually assessed by specialists in CT images. In the trials, the use of tPA was recommended for those patients with an area of ischemia smaller than $1 / 3$ of the MCA $(11,12)$. One of the main side effects of thrombolytic tPA treatment is symptomatic bleeding. This side effect is more marked in patients with a larger infarct. ASPECTS showed that patients with a score equal to or lower than 7 (maximum 10) had a greater chance of bleeding after 
thrombolysis treatment (10). We chose the ASPECTS ROIs because this method has already proved to be of clinical usefulness in guiding treatment and permits coverage of the main structures supplied by the main arteries, in particular the MCA. We added to the ASPECTS ROIs another 3 ROls regarding areas that may be potentially affected by ischemic stroke: the thalamus (in the first slice); part of the medial frontal lobe (anterior portion), supplied by the anterior cerebral artery (in the second slice), and part of the occipital lobe (posterior portion), supplied by the posterior cerebral artery (also in the second slice) (Figure 1). These ROls were placed manually.

Texture parameters for the selected image ROls were computed using the MaZda program (13) developed at the Institute of Electronics, Technical University of Lodz, under the COST project (14). GLC matrices were computed for distances $d=1,2,3,4,5$ pixels, and directions $\theta=0^{\circ}, 45^{\circ}, 90^{\circ}, 135^{\circ}$, for a total of 20 matrices for each ROI. The four texture descriptors aforementioned were calculated for each of these GLC matrices. These parameters were then used to perform statistical discriminant analysis with the Systat 10.2 software, a commercial software from SigmaPlot (www.systat.com).

The statistical analysis was divided into two stages. In the first stage, we investigated the separation of all ROls according to tissue type: lesional tissue, non-lesional tissue from patients and normal tissue from controls. At this stage, no distinction was made between particular anatomical regions. In the second stage, the tissue type discrimination analysis was performed separately for each of the 13 anatomical ROls cited.

After inspection of the results of the first stage analysis, only the parameters calculated for the GLC matrices with $d=1$ and directions $\theta=0^{\circ}$, and $\theta=$ $45^{\circ}$ were found to be significant (the discrimination between tissue types decreased with increasing $d$ ). Therefore, only data from these matrices were used in subsequent analyses.

\section{Results}

We used box plots to visually evaluate the statistical results obtained. These plots are used in descriptive statistics, and they graphically depict groups of numerical data through their five-number summaries: the smallest observation (sample minimum), the lower quartile, the median, the upper quartile, and the largest observation (sample maximum). Box plots may also indicate which observations could be considered outliers; they are useful to determine if there are differences between populations when we cannot make assumptions about the statistical distribution, since they are non-parametric (15). Figures $2 \mathrm{~A}$ and
2B show results of the first-stage discriminant analysis (all ROls considered together), for the ASM and contrast texture parameters, respectively. Since we had 26 ROls (13 for each hemisphere) and 10 subjects ( 5 patients and 5 controls), these
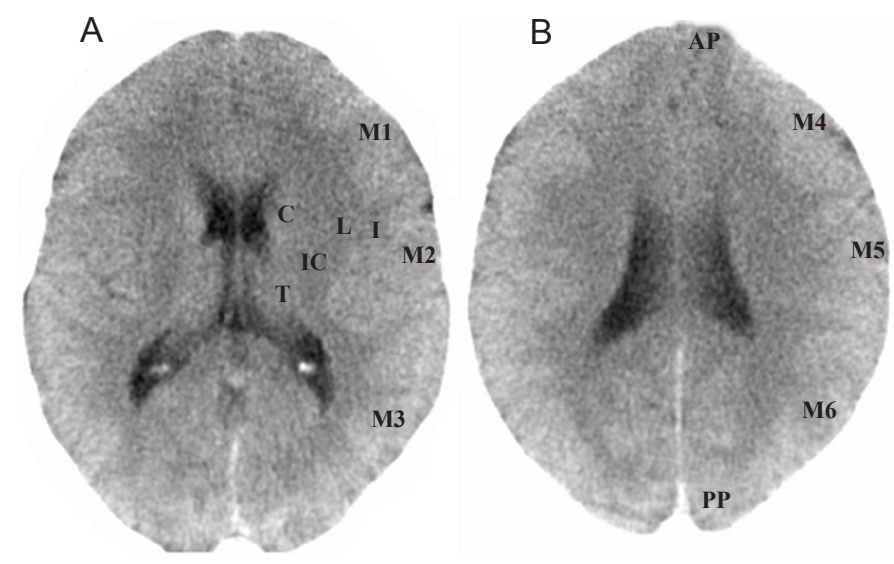

Figure 1. Slices and regions of interest used for texture analysis. $A$, Basal ganglionic axial cut. $B$, Supra ganglionic basal cut. $\mathrm{T}=$ thalamus; $\mathrm{C}=$ caudate; $L$ = lentiform; IC = internal capsule; I = insular ribbon; $M 1, M 2, M 3$, M4, M5, M6 = middle cerebral artery regions; $\mathrm{AP}=$ anterior portion; $\mathrm{PP}=$ posterior portion.
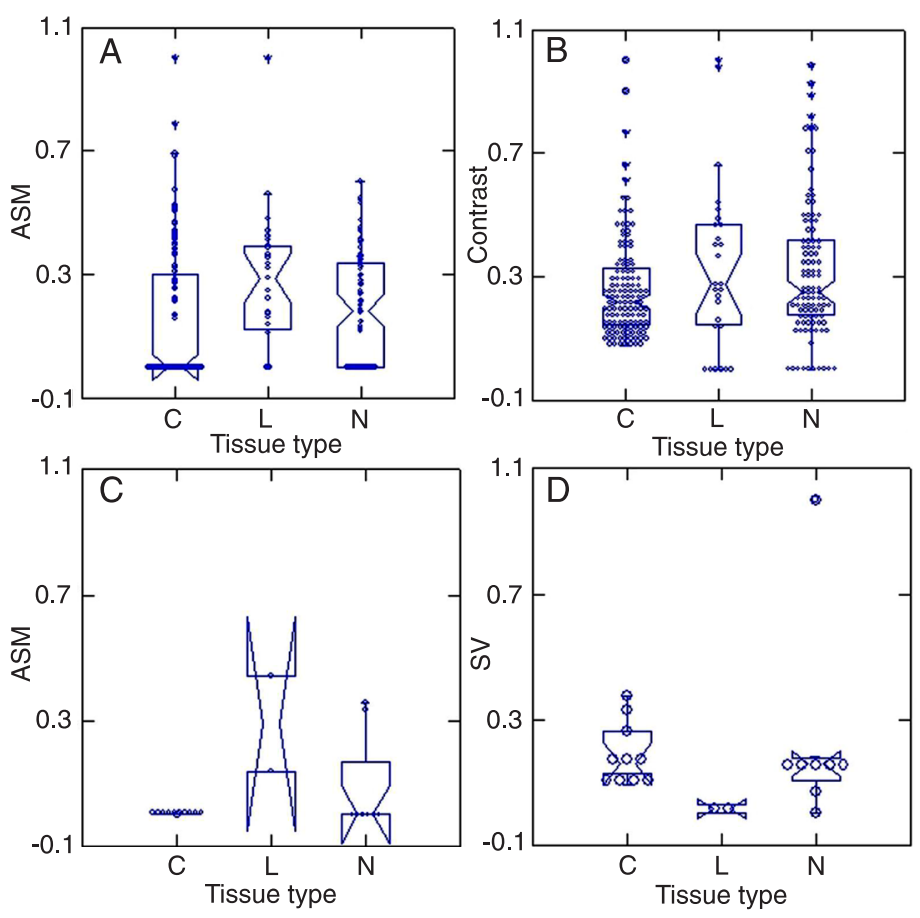

Figure 2. Top, Texture parameters $v s$ tissue type considering all regions of interest (ROIs). Results obtained with the angular second moment (ASM) parameter (A) and the contrast parameter (B). Bottom, Texture parameters vs tissue type for caudate ROI. Results obtained with the ASM parameter (C) and the sum variance (SV) parameter (D). Tissue types are: $\mathrm{C}=$ tissue from controls; $\mathrm{L}=$ lesional tissue from patients; $\mathrm{N}=$ non-lesional tissue from patients. 
plots contain 260 points: $130(13 \times 10)$ points correspond to control ROIs, 102 points to unaltered ROls from patients, and 28 to altered ROIs from patients. Figures $2 \mathrm{C}$ and $2 \mathrm{D}$ show results of the second-stage discriminant analysis for the caudate ROI, for the ASM and for the SV parameters, respectively. In this case, since we had only 2 ROls (caudate in each hemisphere), there were a total of 20 points per plot: 10 points for control ROIs, 8 for unaltered ROls from patients, and 2 for altered ROls from patients.

\section{Discussion}

The plot of ASM against tissue type (Figure 2A) for all ROls clearly shows that there is in fact a distinction, detectable through texture parameters, between controls and patients, and also between the different tissue types (lesional and non-lesional) in patients. The ASM parameter was best at discriminating amongst groups compared to the contrast parameter (Figure 2B), and also compared to the SV and SS parameters (data not shown).

Separate analyses for each ROI showed less discrimination between tissue types, although for some ROls such as the caudate ROI, the ASM was able to provide a clear separation between groups, particularly between control and lesional tis-

\section{References}

1. Tissue plasminogen activator for acute ischemic stroke. The National Institute of Neurological Disorders and Stroke rt-PA Stroke Study Group. N Engl J Med 1995; 333: 1581-1587.

2. The NINDS tPA Stroke Study Group. Generalized efficacy of t-PA for acute stroke: subgroup analysis of the NINDS t-PA stroke trial. Stroke 1997; 28: 2119-2125.

3. Reed SD, Cramer SC, Blough DK, Meyer K, Jarvik JG. Treatment with tissue plasminogen activator and inpatient mortality rates for patients with ischemic stroke treated in community hospitals. Stroke 2001; 32: 1832-1840.

4. Wardlaw JM, Dorman PJ, Lewis SC, Sandercock PA. Can stroke physicians and neuroradiologists identify signs of early cerebral infarction on CT? J Neurol Neurosurg Psychiatry 1999; 67: 651-653.

5. Voigt $\mathrm{H}$. The 'digital eye' at the threshold of cancer diagnosis. Expert Rev Anticancer Ther 2002; 2: 479-480.

6. Haralick RM. Statistical and structural approaches to texture. Proceedings of the IEEE 1979; 67: 786-804.

7. Dougherty ER, Lotufo RA. Hands-on morphological image processing. Bellingham: SPIE Press; 2003.

8. Castellano G, Bonilha L, Li LM, Cendes F. Texture analysis of medical images. Clin Radiol 2004; 59: 1061-1069.

9. Materka A, Strzelecki M. Texture analysis methods - A review. Brussels: Technical University of Lodz, Institute of Electronics, COST B11 REPORT; 1998.

10. Pexman JH, Barber PA, Hill MD, Sevick RJ, Demchuk AM, Hudon ME, et al. Use of the Alberta Stroke Program Early CT Score (ASPECTS) for assessing CT scans in patients sues (Figure 2C). On the other hand, other parameters such as the SV were less discriminating (Figure 2D), but could still show a distinction between lesional and non-lesional tissue in patients. An interesting point is that the SV plot (Figure 2D) showed dispersion for the group considered without alteration. This may suggest the presence of alterations unperceived by visual analysis.

Although these results demonstrate that texture analysis is in fact able to detect tissue alterations in CT images, the manual segmentation of the ROIs can be a limiting factor in the analysis. Future work steps include developing a fully automated analysis method in order to improve the reproducibility of the results, as well as to increase the patients' database.

We presented here the preliminary results of a study under development for the analysis of texture characteristics in CT images of ischemic brain stroke cases. These results showed differences between the texture parameters of control and patient tissues, as well as between lesional and non-lesional tissues from patients. This suggests that the technique may be useful for the detection of regions affected by ischemic stroke. Since the visual analysis of this type of images is usually difficult (16), this could be a promising tool for aiding in the decision process about choosing the best treatment for the patient. with acute stroke. AJNR Am J Neuroradiol 2001; 22: 15341542.

11. Hacke W, Kaste M, Fieschi C, Toni D, Lesaffre E, von Kummer $\mathrm{R}$, et al. Intravenous thrombolysis with recombinant tissue plasminogen activator for acute hemispheric stroke. The European Cooperative Acute Stroke Study (ECASS). JAMA 1995; 274: 1017-1025.

12. Hacke W, Kaste M, Fieschi C, von Kummer R, Davalos A, Meier D, et al. Randomised double-blind placebo-controlled trial of thrombolytic therapy with intravenous alteplase in acute ischaemic stroke (ECASS II). Second EuropeanAustralasian Acute Stroke Study Investigators. Lancet 1998; 352: 1245-1251.

13. Szczypiñski P, Kociolek M, Strzelecki M. MaZda program: computer program for image texture analysis. [Computer program]. http://www.eletel.p.lodz.pl/programy/cost/progr_ mazda_eng.html. Accessed June 3, 2009.

14. COST Project: European Co-operation in the field of Scientific and Technical Research. [Computer program]. http:// www.eletel.p.lodz.pl/programy/cost/project.html. Accessed April 2, 2009.

15. Massart DL, Smeyers-Verbeke J, Caprona X, Schlesier K. Visual presentation of data by means of box plots. LC-GC Europe 2005; 18: 215-218.

16. Weir NU, Pexman JH, Hill MD, Buchan AM. How well does ASPECTS predict the outcome of acute stroke treated with IV tPA? Neurology 2006; 67: 516-518. 